\title{
Nuclear Astrophysics with Tagged Photons
}

\author{
Linda Schnorrenberger*;, Kai Lindenberg, Norbert Pietralla, Deniz Savran, Kerstin \\ Sonnabend \\ Institut für Kernphysik, TU Darmstadt, Germany \\ E-mail: schnorrenberger@ikp.tu-darmstadt.de
}

\section{Michael Elvers, Janis Endres, Jens Hasper, Andreas Zilges}

Institut für Kernphysik, Universität zu Köln, Germany

\begin{abstract}
For the modeling of the astrophysical $s$-process the knowledge of neutron capture cross sections is crucial. Where experimental data is not available for the $(\mathrm{n}, \gamma)$ cross-section like e.g. short-lived branching points, the observation of the inverse $(\gamma, \mathrm{n})$ reaction can provide useful information - in particular, if the cross section can be measured as a function of energy.

The NEPTUN tagger setup at the S-DALINAC delivers a suited tagged photon beam (6-20MeV). In a first test run the resolution turned out to be $35 \mathrm{keV}$ which is close to NEPTUN's design specifications.
\end{abstract}

10th Symposium on Nuclei in the Cosmos

July 27 - August 12008

Mackinac Island, Michigan, USA

\footnotetext{
*Speaker.

${ }^{\dagger}$ Supported by Deutsche Forschungsgemeinschaft under contract SFB 634
} 


\section{Introduction}

Nuclei up to the mass area of iron can be synthesized by fusion reactions like they take place in stars. For heavier nuclei charged-particle cross sections are small even at stellar temperatures. They are mostly produced by neutron capture reactions. One distinguishes mainly two different processes: the rapid neutron capture process (so-called $r$ process) and the slow neutron capture process (so-called $s$ process).

The most favored site for the $r$ process is the explosive scenario of a supernova. Temperatures of about $T \approx 3 \times 10^{9} \mathrm{~K}$ and neutron densities on the order of $n_{n} \gtrsim 10^{20} \mathrm{~cm}^{-3}$ are needed. As long as the neutron flux endures several neutrons are captured before $\beta$ decays occur [1], [2].

On the contrary the $s$ process takes place at lower temperatures along with lower neutron densities. In general, $\beta$ decays take place after the capture of only one neutron if an unstable isotope is reached. The reaction path follows the valley of stability. The $s$ process is classified as follows:

In low mass asymptotic giant branch (AGB) stars the main component is produced. A neutron density of $n_{n} \approx 10^{7} \mathrm{~cm}^{-3}$ at a temperature of $T \approx 9 \times 10^{7} \mathrm{~K}$ is obtained. In the same evolutionary phase recursive He flashes take place along with higher neutron densities of $n_{n} \approx 10^{11} \mathrm{~cm}^{-3}$ and temperatures of $T \approx 2.5 \times 10^{8} \mathrm{~K}$.

The production of the weak component takes place in massive stars during core He-burning $\left(n_{n}<10^{6} \mathrm{~cm}^{-3}, T \approx 3 \times 10^{8} \mathrm{~K}\right)$ and also in the later carbon burning phase $\left(n_{n} \approx 10^{11} \mathrm{~cm}^{-3}\right.$, $\left.T \approx 10^{9} \mathrm{~K}\right)[3]$.

Additionally, about one percent of the nuclei are built by photodissociation of a heavy seed ( $p$ process, see [4]). For some special cases further processes are drawn on.

\section{Motivation}

For a deeper understanding of the nucleosynthesis process large reaction network calculations including all involved nuclei are used to reproduce the observed abundance pattern. The needed cross sections are predicted in theoretical models and to put constraints on the used input parameters as much experimental data as possible is needed.

Most nuclei involved in the s process and undergoing neutron capture are stable and the cross sections are usually well-known. However there are some longer-lived unstable nuclei in the process path. Those are of special interest, because instead of $\beta$ decay an additional neutron capture may occur and lead to a branching of the process path.

For those branching points neutron capture cross sections can only be measured directly in some special cases due to the difficulties with dealing with an unstable target [5], [6], [7]. These experiments showed deviations up to 50\% regarding to recommended values based on [8]. Hence, it is desirable to learn more about cross sections of the branching points. One approach is to study the inverse $(\gamma, n)$ reaction. This has been successfully done in various cases using bremsstrahlung [9], [10]. The drawback of this method is that only energy-integrated cross sections could be determined due to the usage of continuous-energy bremsstrahlung beams.

At facilities with (quasi-)monoenergetic photon beams the cross section can be measured as a function of energy. However, typical energy widths for e.g. Compton backscattering facilities are about $100 \mathrm{keV}$ [11]. The photon tagger system NEPTUN as described below can improve this situation 


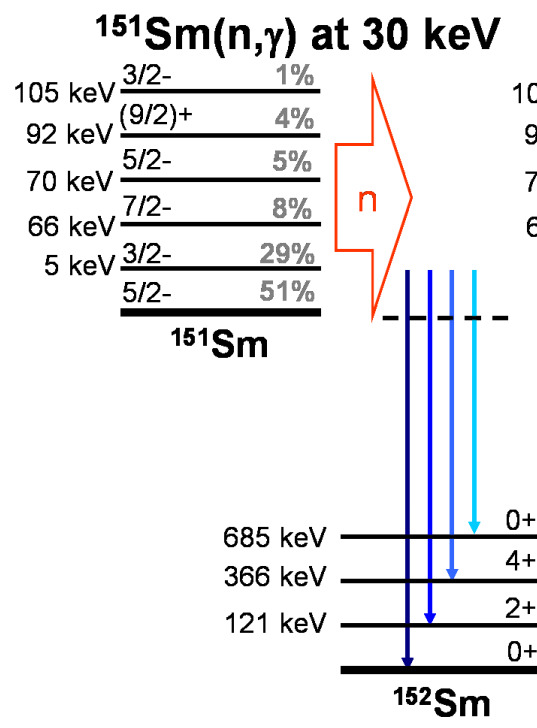

${ }^{152} \mathrm{Sm}(\gamma, \mathrm{n})$ in the lab

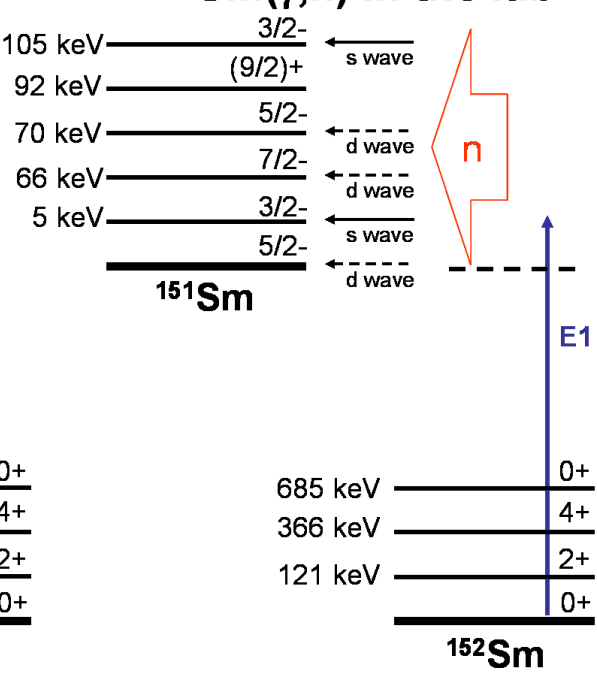

Figure 1: On the left side the level population of ${ }^{151} \mathrm{Sm}$ at a stellar temperature of $T=30 \mathrm{keV}$ is shown. The 5-keV level is occupied at $29 \%$. On the right side the excitation of ${ }^{152} \mathrm{Sm}$ (at lab conditions) by an E1 photon is illustrated. The neutron separation energy of ${ }^{152} \mathrm{Sm}$ is $8257 \mathrm{keV}$. This Figure is based on the calculations presented in [12].

with its energy resolution being better than $35 \mathrm{keV}$.

The convenience of this method will be illustrated for the branching point nucleus ${ }^{151} \mathrm{Sm}$. In this case the ground state neutron capture cross section has been directly measured [7]. However, under stellar conditions, the $5-\mathrm{keV}$ level is occupied at $\approx 30 \%$ and its cross section might differ from extrapolating assumptions (see Fig. 1).

For the inverse $(\gamma, \mathrm{n})$ reaction the specific channel ${ }^{152} \mathrm{Sm}^{\text {g.s. }}(\gamma, \mathrm{n}){ }^{151} \mathrm{Sm}^{5 \mathrm{keV}}$ can be investigated at NEPTUN. Indeed, the ground state cannot be separated from the 5-keV level, but transitions to the ground state are suppressed due to angular momentum selection rules. This will allow to draw conclusions about the $(\mathrm{n}, \gamma)$ cross-section of the first excited state of the branching point ${ }^{151} \mathrm{Sm}$.

\section{The NEPTUN tagger setup}

The basic concept of photon tagging is to select photons of a given energy out of a continuous energy bremsstrahlung spectrum. For bremsstrahlung events conservation of energy yields $E_{\gamma}=E_{0}-E_{e}$ with the photon energy $E_{\gamma}$, the primary beam energy $E_{0}$ and the energy of the scattered electron $E_{e}$. Thus, the photon energy is linearly connected to the energy of the scattered electron. Its momentum can be determined in a spectrometer. By using a coincidence set-up the energy of the photon can be inferred.

The Superconducting Darmstadt Linear Accelerator S-DALINAC delivers the monoenergetic electron beam for the low-energy photon tagger NEPTUN. The beam strikes a thin radiator target right in front of the spectrometer. Typically, a gold target with a thickness of about $10 \mu \mathrm{m}$ is used. Thus, it is guaranteed that $99 \%$ of the electrons produce at most one photon. This ensures the correlation between electron hits in the tagger and photon-induced reactions in the down-stream target.

The electrons which produce bremsstrahlung are scattered into a cone, whereas $99 \%$ are located within an angle of $8^{\circ}$. The magnet's acceptance has been chosen accordingly. To obtain a good energy resolution the electrons have to be focussed in the dispersive plane of a dipole magnet. Additionally a focussing perpendicular to the dispersive plane is necessary to minimize the dimen- 
sions of the focal plane detectors. A Clam-Shell magnet meets these demands. Fringe field effects are reduced by active field clamps.

The focal plane detector array is built of 32 scintillating fibers attached to photomultipliers. The profile of the fibers is quadratic and the width has been chosen to $1 \mathrm{~mm}$ to exceed the resolution power of the magnet. An upgrade to 128 fibers is in progress. This will enable to measure a larger energy window in one run without a change of the magnetic field. At a photon energy of $\mathrm{E}_{\gamma}=10 \mathrm{MeV}$, the range will be about $\Delta \mathrm{E}_{\gamma}=1-3 \mathrm{MeV}$ depending on the arrangement of the 128 fibers. NEPTUN has been designed to tag photons in the energy range from $6-20 \mathrm{MeV}$.

The produced photons pass a copper collimator ( $40 \mathrm{~cm}$ thick) embedded in a shielding concrete wall ( $\approx 1 \mathrm{~m}$ thick). This benefits the placement of detectors close to the experimental target position at low-background conditions. The expected tagged photon flux at the target position is about $0.5 \times 10^{3}$ photons $\mathrm{keV}^{-1} \mathrm{~cm}^{-2} \mathrm{~s}^{-1}$. For more details see [13] and [14].

For $\left(\gamma, \gamma^{\prime}\right)$ experiments High-Purity Germanium Detectors are available. A neutron detector array is built for the use in $(\gamma, n)$ experiments. The data acquisition system is based on VME. Its core is a 128 channel multi-hit TDC, which is able to connect hits within a time window of $4 \mu \mathrm{s}$. This is mandatory to assign electron hits to slow neutron events like they occur in experiments close to the particle threshold where the kinetic energy of the neutrons is low.

\section{Test Experiments}

The tagging quality of NEPTUN has been tested by placing a HPGe detector directly at the experimental target position in order to measure the energy profile of the photon beam. By gating on one scintillating fiber (= a specific focal plane position) the photon energy could be chosen. The resulting spectrum is reduced to the typical detector response of a HPGe on a monoenergetic photon beam. This could be confirmed by GEANT4 simulations. The energy resolution is better than $35 \mathrm{keV}$, which corresponds to the energy spread of the electron beam in this experiment (see Fig. 2). Further improvement on the energy spread of the accelerator is in progress.

It could also be shown that even at high beam currents, the background around the experimental target position is negligible. Detectors can be placed as close as about $10 \mathrm{~cm}$ to the target position which helps to cover large solid angles.
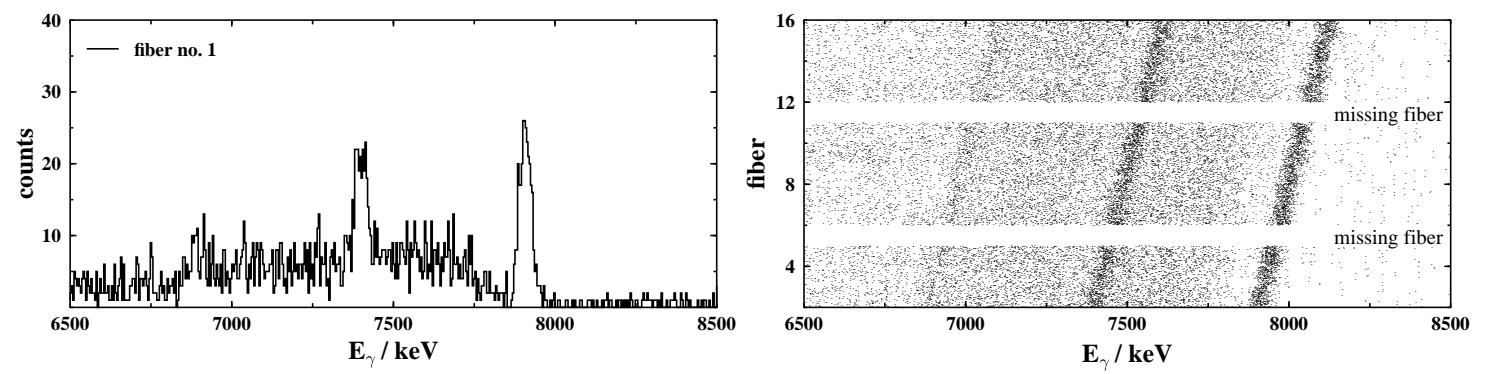

Figure 2: Left panel: Spectrum of a HPGe detector placed in the photon beam gated on one focal plane position, i.e. one fiber. Right panel: Same setup as left panel showing focal plane position (fiber number) vs. energy in the HPGe as a scatter plot (horizontal white lines are due to missing fibers). Full energy peak, Compton edge, single and double escape lines can easily be distinguished. The energy resolution achieved so far is better than $35 \mathrm{keV}$. 


\section{Conclusions and Outlook}

The high resolution low-energy photon tagger NEPTUN is a powerful tool for nuclear astrophysics. As an example the $s$ process branching point ${ }^{151} \mathrm{Sm}$ has been discussed.

A first test run demonstrated a $35-\mathrm{keV}$ resolution of NEPTUN which is close to the design specification of $25 \mathrm{keV}$. An even better resolution should be gained by improvements on the side of the accelerator.

The upgrade of the focal plane detector array from 32 to 128 fibers is in progress and will lead to a larger energy window to be measured at one setting of the magnetic field.

Further, the construction of a neutron ball consisting of liquid scintillators has been started.

Besides standard liquid scintillators also ${ }^{10} \mathrm{~B}$-loaded ones will be used to allow the discrimination of photons against neutrons down to the lowest neutron energies.

\section{References}

[1] K.-L. Kratz, J.-P. Bitouzet, F.-K. Thielemann, P. Möller, B. Pfeiffer, Astrophys. J. 403 (1993) 216.

[2] G. Wallerstein, I. Iben, P. Parker, A. M. Boesgaard, G. M. Hale, A. E. Champagne, C. A. Barnes, F. Käppeler, V. V. Smith, R. D. Hoffman, F. X. Timmes, C. Sneden, R. N. Boyd, B. S. Meyer, D. L. Lambert, Rev. Mod. Phys. 69 (1997) 995.

[3] F. Käppeler, A. Mengoni, Nucl. Phys. A777 (2006) 291.

[4] M. Arnould, S. Goriely, Phys. Rep. 384 (2003) 1.

[5] S. Jaag, F. Käppeler, Phys. Rev. C 51 (1995) 3465.

[6] R. Reifarth, C. Arlandini, M. Heil, F. Käppeler, P. V. Sedyshev, A. Mengoni, M. Herman, T. Rauscher, R. Gallino, Astrophys. J. 582 (2003) 1251.

[7] K. Wisshak, F. Voss, F. Käppeler, Phys. Rev. C 73 (2006) 015802.

[8] Z. Y. Bao, H. Beer, F. Käppeler, F. Voss, K. Wisshak, T. Rauscher, At. Data Nucl. Data Tables 76 (2000) 70 .

[9] K. Sonnabend, P. Mohr, K. Vogt, A. Zilges, A. Mengoni, T. Rauscher, H. Beer, F. Käppeler,

R. Gallino, Astrophys. J. 583 (2003) 506.

[10] K. Sonnabend, A. Mengoni, P. Mohr, T. Rauscher, K. Vogt, A. Zilges, in M. Arnould et al., Ed., Proceedings of the Conference Tours Symposium on Nuclear Physics V, 2003, AIP Conf. Proc. 2004, (p. 463).

[11] H. R. Weller, M. W. Ahmed, Mod. Phys. Lett. A 23 (2003) 1569.

[12] A. Mengoni, in AIP Conf. Proc. 769: International Conference on Nuclear Data for Science and Technology, 2005, (p. 1209).

[13] K. Lindenberg, Development and Construction of the Low-Energy Photon Tagger NEPTUN, PhD Thesis, Technische Universität Darmstadt, 2008.

[14] M. Elvers, J. Hasper, S. Müller, D. Savran, L. Schnorrenberger, K. Sonnabend, A. Zilges, J. Phys. G 35 (2008) 014027. 